\title{
Structural and Compositional Evolution of Self-Assembled Germanium Islands on Silicon (001) During High Growth Rate LPCVD
}

\author{
Gabriela D.M. Dilliway ${ }^{1}$, Nicholas E.B. Cowern ${ }^{2}$, Chris Jeynes ${ }^{2}$, Lisa O’Reilly ${ }^{3}$, Patrick J. \\ McNally $^{3}$, Darren M. Bagnall ${ }^{1}$ \\ ${ }^{1}$ Dept. of Electronics and Computer Science, Univ. of Southampton, Highfield, Southampton \\ SO17 1BJ, UK \\ ${ }^{2}$ Advanced Technology Institute, Univ. of Surrey, Guildford GU2 7XH, UK \\ ${ }^{3}$ Research Institute for Networks \& Communications Engineering (RINCE), School of \\ Electronic Engineering, Dublin City University, Dublin 9, Ireland
}

\begin{abstract}
Understanding the process of self-organization of Ge nanostructures on Si with controlled size distribution is a key requirement for their application to devices. In this study, we investigate the temporal evolution of self-assembled islands during the low pressure chemical vapour deposition (LPCVD) of $\mathrm{Ge}$ on $\mathrm{Si}$ at $650^{\circ} \mathrm{C}$ using high growth rates $(6-9 \mathrm{~nm} / \mathrm{min})$. The islands were characterized by atomic force microscopy, transmission electron microscopy, Rutherford backscattering spectrometry and micro-Raman spectroscopy. We found that the first nanostructures to assemble were small islands, with a narrow size distribution, typical of the 'lens-shaped' structures reported in previous studies. Next to form were a population of larger 'lens-shaped' islands with a similar surface density to that of the small islands, but with broad height and width distributions. These islands differ from the pyramid-shaped islands previously reported for a similar size range. On further Ge deposition, the population evolves into one of large square-based truncated pyramids with a very narrow size distribution. Such pyramidal structures were previously reported at smaller sizes. Furthermore, we see no evidence of the multifaceted domes previously reported in this size range. The small 'lens-shaped' islands appear to be strained, whilst some of the intermediate-sized islands and all the large truncated pyramids contain misfit strain relaxation induced defects. Additionally, in the both the intermediate size 'lens-shaped' islands and in the large size truncated pyramidal islands, there is evidence of Si-Ge strain-induced alloying, more significant in the first than in the latter. Our observation of 'lens shaped' islands and truncated pyramids at larger sizes than are normally observed, suggests a kinetically driven process that delays the evolution of energetically favourable island structures until larger island sizes are reached.
\end{abstract}

\section{INTRODUCTION}

In recent years, substantial efforts have been devoted to research into quantum dot structures, due to the interesting electronic and optical properties that can result from quantum confinement effects. Obtaining quantum structures through self-organisation has the advantage of enabling fabrication of electronic and optical devices of dimensions smaller than accessible by lithography. The process of self-assembly of Ge nanoscale islands is of particular interest because of its compatibility with Si-based technology. However, this process still has limitations that need to be overcome, e.g. the placement of the individual self-assembled structures cannot be defined at the beginning of growth and island sizes may be distributed over a wide range.

Qualitatively, Si and Ge are very similar in their electronic and structural properties. They both crystallise in a diamond cubic structure, but the lattice constant of $\mathrm{Ge}$ is larger than that of 
$\mathrm{Si}$, by $\sim 4.2 \%$, resulting in a Stranski-Krastanow growth mode for $\mathrm{Ge}$ on $\mathrm{Si}$. This starts as layerby-layer or two-dimensional growth and proceeds as clustering or three-dimensional growth. Because of its larger lattice constant, Ge stores a high strain energy density as it grows on a Si substrate. As a consequence, it has been observed that after about 3 monolayers (ML) of Ge have been deposited on $\mathrm{Si}$, the growing surface becomes non-planar and islands form, thus relieving part of the misfit strain through lateral expansion of the Ge lattice at the island peak [1]. It is hoped that a better understanding of the mechanisms involved in Ge island growth will lead to the discovery of methods to control their distribution and size. A significant number of studies have been performed with the aim of understanding the structural and compositional evolution of Ge self-assembled islands grown on (001) Si substrates. The role played by different growth parameters, such as temperature, time, pressure, growth rate and others have been studied for different growth techniques. We are aiming to understand the evolution of such islands during LPCVD with high growth rates $(6-9 \mathrm{~nm} / \mathrm{min})$.

In this paper we report results obtained on the evolution of Ge self-assembled islands grown under nominally identical conditions, for three different durations, in an LPCVD) reactor designed and fabricated at the Southampton University Microelectronics Centre (SUMC). The growth rates used in this study $(6-9 \mathrm{~nm} / \mathrm{min})$ are higher than have generally been reported up to now. Under our growth conditions, a population of intermediate size 'lens-shaped' islands evolves rapidly into a population of truncated pyramidal islands with a lower island density and a very narrow size distribution. This evolution differs from that found in previous studies [2-5], where pyramidal structures were observed to form at intermediate sizes $(\sim 150 \mathrm{~nm})$ and subsequently evolve into multi-faceted domes, and finally into larger stable pyramids.

\section{EXPERIMENTAL TECHNIQUES}

The LPCVD reactor used for the growth of the samples is an experimental, cold-wall, single wafer system. It works at growth pressures up to 1 Torr, thus higher than those used in ultra high vacuum systems such as ultra high vacuum CVD and molecular beam epitaxy, and in other LPCVD systems reported in the literature (0.5-2 mTorr [2] and $120 \mathrm{mTorr}[6,7])$, but lower than those used in atmospheric-pressure CVD. The pressure chosen for the growth of the Ge nanostructures was 0.5 Torr. The growth technique used allows control over both the growth pressure and the source gas flow, thus enabling additional control over the growth rates.

For this study, a set of three samples were grown and subsequently analysed. $100 \mathrm{~mm}(001)$ n-type $\mathrm{Si}$ wafers were subjected to an ex-situ standard RCA-clean and the 'RCA' oxide was subsequently removed in-situ by a $\mathrm{H}_{2}$ bake at $950^{\circ} \mathrm{C}$. The temperature was then lowered to $900^{\circ} \mathrm{C}$ in $\mathrm{H}_{2}$ flow and a $500 \mathrm{~nm}$ thick Si buffer was grown from $\mathrm{SiH}_{4}(100 \%)$ at 1 Torr, to ensure an inhomogeneity-free growth surface. Finally, the temperature was lowered to $650^{\circ} \mathrm{C}$ in $\mathrm{H}_{2}$ flow, the base pressure was reduced to 0.5 Torr, and $\mathrm{GeH}_{4}(10.2 \%$ in hydrogen) was flushed in the growth chamber. The process was repeated on three different wafers for three different durations, resulting in the formation of Ge islands ranging from a few tens of $\mathrm{nm}$ in width and under $4 \mathrm{~nm}$ in height for the shortest growth time, up to $250 \mathrm{~nm}$ width and $50 \mathrm{~nm}$ height for the longest growth time. Samples grown were subsequently analysed by atomic force microscopy (AFM), cross-sectional transmission electron microscopy (XTEM), Rutherford backscattering spectroscopy (RBS) and micro-Raman spectroscopy.

A TopoMetrix Accurex II ${ }^{\mathrm{TM}}$ scanning probe microscope in contact mode was used for the analysis of the surface morphology of the three samples. This technique provided information on the size and height distribution and on the density of the Ge self-assembled islands. 
A J.E.O.L. JEM 2000FX TEM with a $\mathrm{LaB}_{6}$ crystal beam source was used in order to assess the state of strain relaxation of the Ge islands, through their crystalline quality.

Further insight into the crystalline quality and compositional evolution of the Ge islands was obtained by detailed analysis of RBS and micro-Raman spectra. For the RBS studies, the samples were probed with a $2 \mathrm{MeV}$ He analysing beam at normal incidence and the scattered He ions were detected by a solid-state detector, with a resolution energy of $15 \mathrm{keV}$. Both 'random' and $<100>$ channelling directions were used in order to analyse the composition and crystalline quality of the islands. Micro-Raman analyses were performed on a Jobin-Yvon LabRam800 system, used in backscattering configuration in which only the longitudinal optical modes are activated for both the substrate and the epitaxial layers. A $488 \mathrm{~nm} \mathrm{Ar}^{+}$laser with a spot size of $1 \mu \mathrm{m}$ which probes right through to the Si substrate was used and three different positions on each sample were scanned for 5 min each. Longer scans (of 15 min) were also carried out for control purposes.

\section{RESULTS AND DISCUSSION}

The amount of Ge deposited on the three wafers was given by RBS measurements in 'random' orientation and is expressed here in terms of equivalent monolayers (ML) of Ge on the surface. For consistency with the literature, the lattice constant of bulk Ge is used, hence one equivalent monolayer is $\sim 6.3 \times 10^{14} \mathrm{Ge}$ atoms $\mathrm{cm}^{-2}$. RBS results showed a Ge thickness (averaged over the beam-spot area of $\approx 1 \mathrm{~mm}^{2}$, which includes a large number of islands and the surface between them) of $\sim 4 \mathrm{ML}$ in the shortest growth duration wafer, $\sim 6 \mathrm{ML}$ in the medium duration wafer, and $\sim 11 \mathrm{ML}$ in the longest duration wafer. The amount of Ge deposited increases linearly with the growth duration indicating good growth control. Analysis in $<100>$ channelling mode showed values of $\chi_{\min }$ of $3.8 \%$ for the $4 \mathrm{ML}$ sample, $5.0 \%$ for the $6 \mathrm{ML}$ sample, and $4.9 \%$ for the $11 \mathrm{ML}$ sample. These values indicate a low level or absence of defects in the islands of the shortest duration wafer, and the presence of defects in the islands of the intermediate and longest duration wafers.

AFM results obtained on $5 \times 5 \mathrm{~m}^{2}$ scanned area and shown in figure 1 (3D images) provided values for the density of Ge islands in all three samples studied. Line analyses carried out on $1 \times 1 \mu \mathrm{m}^{2}$ AFM scanned areas allowed the measurement of the width and height distribution of the Ge self-assembled islands in all three samples studied (figure 2).

In the case of $4 \mathrm{ML}$ of deposited Ge, we find small 'lens-shaped' islands with a surface density of $\sim 16 \mu \mathrm{m}^{-2}$ and a rather narrow size distribution, ranging from 20-30 $\mathrm{nm}$ in width and 2$4 \mathrm{~nm}$ in height (figures $1 \mathrm{a}$ and $2 \mathrm{a}$ ). In the case of $6 \mathrm{ML}$ of deposited Ge, just $2 \mathrm{ML}$ more than in case (a), a mixed population of small and larger 'lens-shaped' islands has developed (figures $1 \mathrm{~b}$ and $2 b)$. The total density $\left(\sim 19 \mu \mathrm{m}^{-2}\right)$ has not changed significantly from the previous case, suggesting that growth, rather than nucleation or ripening, is the dominant process at this stage. Most islands are between 100-150 nm in width and 10-25 nm in height, and XTEM analysis reveals strain relaxation induced defects within the islands (arrows in figure 3a). This observation is consistent with RBS results, which show a higher level of dechannelling in this sample than in the previous one. A dramatically different situation holds in the case of $11 \mathrm{ML}$ of deposited Ge, in which a further $5 \mathrm{ML}$ of Ge have been deposited. In this case, the density of islands has dropped by almost one order of magnitude $\left(\sim 3 \mu \mathrm{m}^{-2}\right)$, both the small and medium size 'lens-shaped' islands have disappeared, and have been replaced by a population of large squarebased truncated pyramids (figures $1 \mathrm{c}$ and $2 \mathrm{c}$ ). 


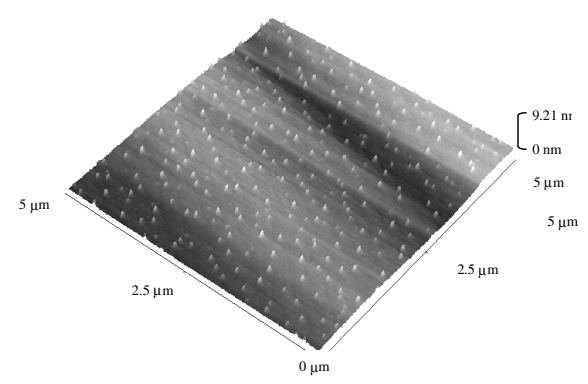

(a)

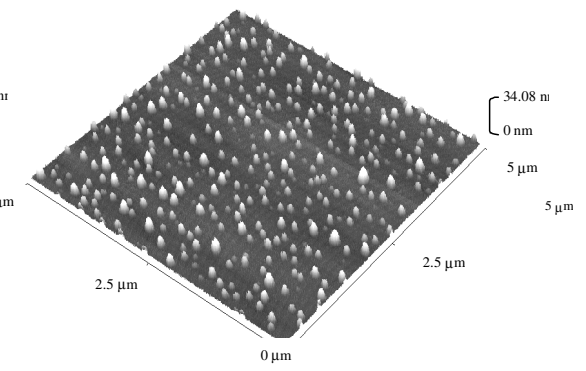

(b)

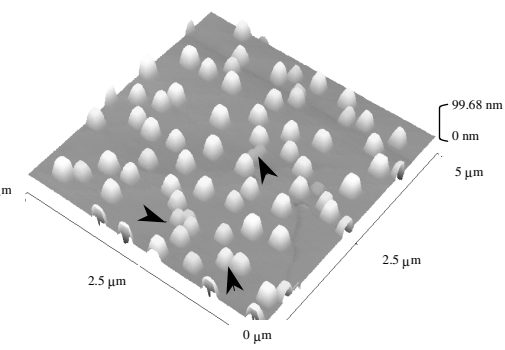

(c)

Figure 1. AFM images of $5 \times 5 \mathrm{~m}^{2}$ scanned areas after the deposition of: (a) $4 \mathrm{ML}$; (b) $6 \mathrm{ML}$; (c) $11 \mathrm{ML}$ of Ge on Si.

This rapid transition indicates that the energy of the system has decreased drastically on conversion to truncated pyramidal islands. The size distribution of the majority of these islands is very narrow ( $250 \mathrm{~nm}$ wide) and the height of the islands has increased substantially ( $\sim 50 \mathrm{~nm})$. The few islands that remain within the intermediate size range have converted to truncated pyramids, but the growth of each of them has been restricted by its proximity to another island (arrows in figure 1c). It seems likely that these islands mark the size at which the transformation into truncated pyramids starts to occur, and that the size of the majority of isolated islands is a result of (i) further Ge deposition and (ii) the consumption of Ge from the previously existing lens-shaped islands. XTEM analysis of the vicinal islands shows an asymmetric configuration, in which one side of the islands forms a (111) facet, characteristic of truncated pyramidal islands (figure $3 \mathrm{~b}$ ). In most of the large isolated islands a significant density of misfit dislocations can be seen (indicated by the arrows in figure $3 \mathrm{c}$ ). This observation is, once again, consistent with RBS results, which show a significantly high level of dechannelling in this sample.

Micro Raman results showed that the two most important Si-Si peaks, located at $520 \mathrm{~cm}^{-1}$ and $300 \mathrm{~cm}^{-1}$ are visible in all three samples studied. This result was obtained by comparing spectra for the analysed samples with a Si control spectrum. Results obtained for the sample grown for the shortest duration (not shown here) lead us to conclude that the technique used cannot resolve the very small Ge islands present in this sample.

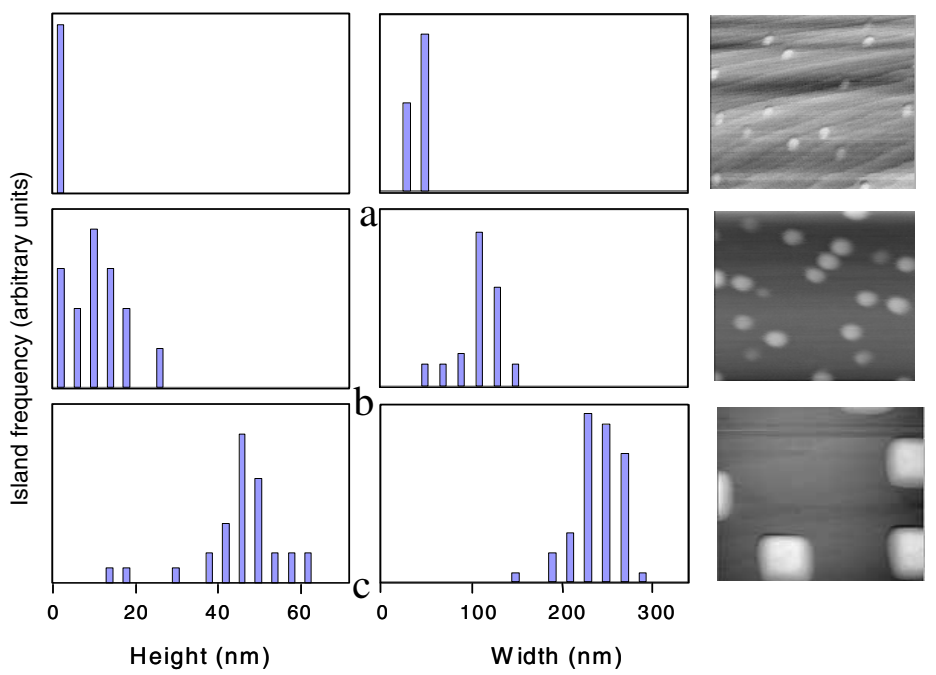

Figure 2. Height and width distributions together with $2 \mathrm{D}$ AFM images of $1 \mathrm{x} 1 \mu \mathrm{m}^{2}$ scanned areas of: (a) the shortest growth duration sample; (b) the intermediate growth duration sample; (c) the longest growth duration sample. 


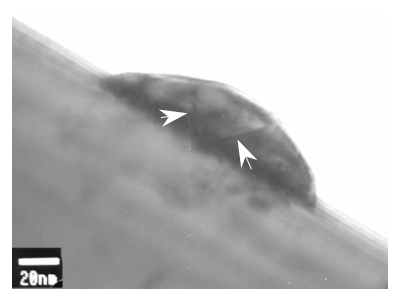

(a)

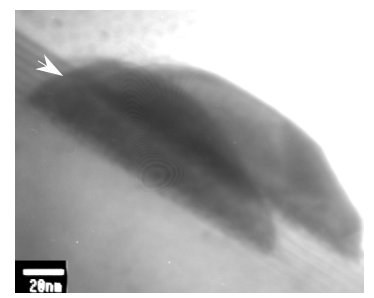

(b)

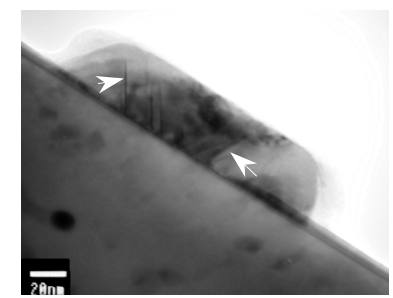

(c)

Figure 3. Bright-field XTEM micrographs of: (a) the intermediate growth duration sample, showing a dislocated 'lens-shaped' Ge island; (b) the longest growth duration sample, showing two asymmetrical medium-sized islands; (c) the longest growth duration sample, showing a large dislocated truncated pyramidal island.

In the case of the intermediate and long growth duration samples, in order to enable a more detailed analysis of the interaction between the Ge layer and the Si epilayer, the Si background was removed. The micro-Raman results thus obtained show two peaks of interest (figure 4): one at $297 \mathrm{~cm}^{-1}$, which although close to the Si-Si peak at $300 \mathrm{~cm}^{-1}$, is in fact attributable to a GeGe mode [8-11] and one at $\sim 400 \mathrm{~cm}^{-1}$, which is attributable to a Si-Ge mode [11-12]. Based on the relative intensities of the $\mathrm{Si}-\mathrm{Ge}$ and $\mathrm{Ge}-\mathrm{Ge}$ peaks, the average content of the islands can be obtained according to the relation: $\mathrm{I}_{\mathrm{Si}-\mathrm{Ge}} / \mathrm{I}_{\mathrm{Ge}-\mathrm{Ge}}=2(1-\mathrm{x}) / \mathrm{x}$, where $\mathrm{x}$ is the Ge composition [11-12]. An average Ge fraction of $\sim 70 \%$ can be found in the $6 \mathrm{ML}$ sample, and of $\sim 90 \%$ in the $11 \mathrm{ML}$ sample. This estimation neglects quantum confinement effects. The Si-Ge peak in the case of the large truncated pyramidal islands is weaker and broader than in the case of the medium-sized 'lens-shaped' islands and is shifted to lower wavenumbers (i.e. $\sim 411 \mathrm{~cm}^{-1}$ compared with $\sim 394 \mathrm{~cm}^{-1}$ ). The lower Si-Ge intensity implies less intermixing, and the larger broadening indicates a greater dislocation density in the large islands, as already established by XTEM (figure $3 \mathrm{c}$ ).

A small 'shoulder' at $\sim 470 \mathrm{~cm}^{-1}$, seen on the spectrum for the intermediate growth duration sample (indicated by the arrow in figure 4), is attributable to a downward shift in the Si-Si mode due to intermixing with Ge. The magnitude of the shift, about $50 \mathrm{~cm}^{-1}$, is about $70 \%$ of the expected shift per unit Ge composition, i.e. it is consistent with our previous estimate that the islands have $\sim 70 \%$ Ge content. The absence of a shoulder in the spectrum for the longest duration sample indicates a much smaller amount of intermixing, as already established from the much weaker Si-Ge signal in that sample.

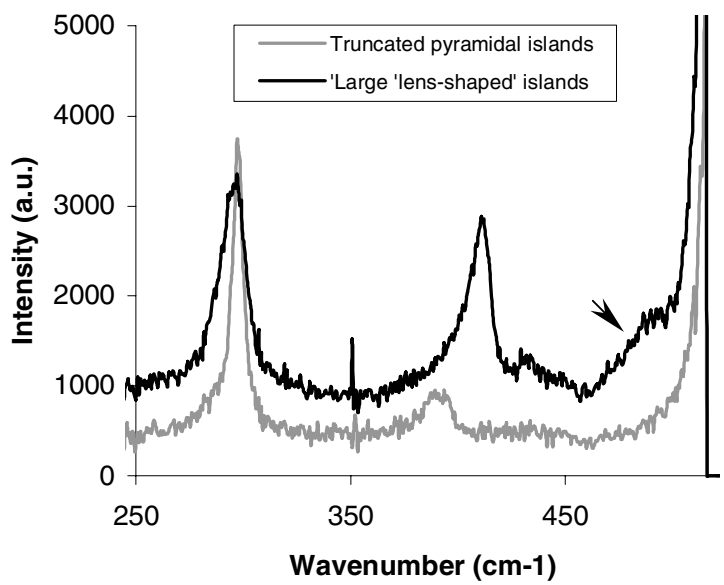

Figure 4. Micro-Raman spectra for the intermediate growth duration sample (the dark arrow points to a 'shoulder' corresponding to the $\mathrm{Si}-\mathrm{Si}$ mode peak of the epilayer and for the long growth duration sample. 
It is interesting to speculate as to why we observe such structures as 'lens-shaped' and truncated pyramids at large island sizes, rather than the dome-shaped and full pyramidal islands usually observed at these sizes. We suspect the reason is that the high growth rate used prevents the reorganization of the island structure within the time frame available as the islands grow through each size regime. This is typical of a process that is kinetically driven, and far from thermodynamic equilibrium. The strong interdiffusion in the medium sized 'lens-shaped' islands, and the accompanying presence of relaxation defects, attest to the high driving force for structural change, while the relatively low intermixing in the larger islands shows that the system has approached closer to equilibrium, even though no transition has occurred to dome-shaped or full pyramidal structures.

\section{CONCLUSIONS}

Our observation of 'lens-shaped' islands and truncated pyramids at larger sizes than have normally been reported, suggests a kinetically driven process that delays the evolution of energetically preferred structures until larger sizes are reached. Analysis with a range of experimental techniques has revealed the mechanisms through which the islands formed at high growth rates 'attempt' to approach lower energy configurations. These include the simultaneous occurrence of interdiffusion and defect formation. An abrupt transition from 'lens-shaped' to truncated pyramidal islands at a deposited Ge level of about 11 ML leads to a reduction in strain energy through a more ordered structural arrangement, and an island composition which is closer to pure Ge. The size distribution of the resultant islands is unusually narrow, apart from a small population of vicinal islands which are unable to grow due to overlap with a neighbouring island. If placement of the islands could also be controlled to prevent overlap, it is likely that the resulting islands would have a perfectly uniform size distribution.

\section{REFERENCES}

1. F.M. Ross, IBM Res. Develop. 44, 489 (2000).

2. F. Boscherini, G. Capellini, L. di Gaspare, F. Rosei, N. Motta, S. Mobilio, Appl. Phys. Lett. 76, 682 (2000).

3. T.I. Kamins, G. Medeiros-Ribeiro, D.A.A. Ohlberg, R. Stanley Williams, Appl. Phys. A 67, 727 (1998).

4. X.Z. Liao, J. Zou, D.J.H. Cockayne, Z.M. Jiang, X. Wang and R. Leon, Appl. Phys. Lett. 77, 1304 (2000).

5. R. Loo, P. Meunier-Beillard, D. Vanhaeren, H. Bender, M. Caymax, W. Vandervorst, D. Dentel, M. Goryll, L. Vescan, J. Appl. Phys. 90, 2565 (2001).

6. M. Goryll, L. Vescan, K. Schmidt, S. Mesters, H. Lüth, Appl. Phys. Lett. 71, 410 (1997).

7. L. Vescan, T. Stoica, O. Chretien, M. Goryll, E. Mateeva, A. Mück, J. Appl. Phys. 87, 7275 (2000).

8. G.B Wright et al., Phys. Rev. Lett., 18, 607 (1967).

9. T. Kobayashi, T. Endoh, H. Fukuda, S. Noamura, A. Sakai, Y. Ueda, Appl. Phys. Lett., 71, 1195 (1997).

10. X.L. Wu, T. Gao, X.M. Bao, F. Yan, S.S. Jiang, D. Feng, J. Appl. Phys., 82, 2704 (1997).

11. A. Eshed, J. Zhu, J. Yan, R. Beserman, A.H. Weiss, Mat. Res. Soc. Symp. Proc., 696, N.3.5.1 (2002).

12. P.M. Mooney, F.H. Dacol, J.C. Tsang and J.O. Chu, Appl. Phys. Lett., 62, 2069 (1993). 